\title{
Incidencia de la Contaminación por Metales Ecotóxicos en la Fauna Marina Bentónica en la Bahía de Ite, Provincia de Jorge Basadre Grohmann de Tacna
}

\section{Pollution Incidence with Eco toxic Metals in the Benthic Marine Fauna in Ite Bay, Jorge Basadre Grohmann Province of Tacna}

\section{${ }^{1}$ Lorenzo Walter Ibárcena Fernández}

\begin{abstract}
RESUMEN:
En el presente trabajo se hace un estudio de los niveles de contaminación por elementos ecotóxicos en sedimentos marinos por efectos antropogénicos, de los siguiente elementos: $\mathrm{Cu}, \mathrm{Zn}, \mathrm{Cd}, \mathrm{As}, \mathrm{Hg}, \mathrm{Pby} \mathrm{Fe}$, en la Bahía de Ite, Distrito de Ite, Provincia de Jorge Basadre Grohmann de Tacna.

La cuantificación de la fauna marina bentónica de la zona fue realizada, para poder efectuar comparaciones con los estándares existentes para zonas afectadas por efectos antropogénicos, y establecer si se ha generado daño ecológico por la acumulación de los relaves mineros, y en qué medida ha sido afectada la fauna marina bentónica.

También se efectuaron análisis de humedad, materia orgánica y potencial de hidrógeno de los sedimentos en las nueve estaciones de muestreo en la zona de estudio (Bahia de Ite).

Las condiciones oceanográficas como temperatura, salinidad, oxigeno disuelto y transparencia en el agua marina, también fueron consideradas para establecer si existe diferencia entre los valores obtenidos en nuestros análisis, con valores estándares del litoral sur del Perú, reportados por el Instituto del Mar del Perú, Estación Costera de Ilo.
\end{abstract}

Palabras clave: contaminación, ecotóxicos, sedimentos marinos.

\section{ABSTRACT:}

This paper presents a study of pollution levels in marine sediments by ecotoxic elements originated by anthropogenic effects, such elements are: $\mathrm{Cu}, \mathrm{Zn}, \mathrm{Cd}, \mathrm{As}, \mathrm{Hg}, \mathrm{Pb}$ and $\mathrm{Fe}$; their presence in the Bay of Ite, District of Ite, Jorge Basadre Grohmann province of Tacna was analyzed.

The quantification of the benthic marine fauna of the area was conducted in order to make comparisons with existing standards for areas affected by anthropogenic effects, and to determine whether in the area some environmental damage has been generated by the accumulation of mine tailings, and in what way benthic marine fauna has been affected.

Also analysis of moisture, organic matter and hydrogen potential of sediments in the nine sampling stations in the study area (Bay of Ite) were conducted.

Oceanographic conditions such as temperature, salinity, dissolved oxygen and transparency in seawater were also considered to establish if there are differences between values obtained in our analysis and standard values of the southern coast of Peru, reported by the Instituto del Mar del Peru, Laboratorio costero, Ilo.

Keywords: pollution, Eco toxic, marine sediments Doctor en Ciencias y Tecnologias Medioambientales, Maestro en Ciencias con Mención en Computación e Informática, Ingeniero Pesquero.
Facultad de Ciencias Agropecuarias. Universidad Nacional Jorge Basadre Grohmann. 


\section{INTRODUCCIÓN}

Uno de los problemas ambientales que han suscitado mayor interés mundial en esta década es la pérdida de biodiversidad como consecuencia de las actividades humanas, ya sea de manera directa (sobreexplotación) o indirecta (alteración del hábitat). Los medios de comunicación han impactado de tal manera que tanto el gobierno, la iniciativa privada, como la sociedad en general consideran prioritario dirigir mayores esfuerzos hacia programas de conservación. La base para un análisis objetivo de la biodiversidad y su cambio reside en su correcta evaluación y monitoreo.

La biodiversidad o diversidad biológica se define como "la variabilidad entre los organismos vivientes de todas las fuentes, incluyendo, entre otros, los organismos terrestres, marinos y de otros ecosistemas acuáticos, así como los complejos ecológicos de los que forman parte; esto incluye diversidad dentro de las especies, entre especies y de ecosistemas" (UNEP, 1992). El término comprende, por tanto, diferentes escalas biológicas: desde la variabilidad en el contenido genético de los individuos y las poblaciones, el conjunto de especies que integran grupos funcionales y comunidades completas, hasta el conjunto de comunidades de un paisaje o región (Solbrig, 1991; Halffter y Ezcurra, 1992; Heywood, 1994).

La diversidad biológica representa un tema central de la teoría ecológica y ha sido objeto de amplio debate (Magurran, 1988). La falta de definición y de parámetros adecuados para su medición hasta principios de los 70's llevó incluso a declarar la falta de validez del concepto (Hurlbert, 1971). Actualmente el significado y la importancia de la biodiversidad no están en duda y se han desarrollado una gran cantidad de parámetros para medirla como un indicador del estado de los sistemas ecológicos, con aplicabilidad práctica para fines de conservación, manejo y monitoreo ambiental (Spellerberg, 1991). Por lo indicado nos plantemos los siguientes objetivos:

\section{Objetivo General}

Determinar la incidencia de la contaminación por metales ecotóxicos en la fauna marina bentónica en la Bahía de Ite, Provincia de Jorge Basadre Grohmann de Tacna

\section{Objetivos Específicos:}

$\Lambda$

Determinar la perturbación generada por la contaminación de metales ecotóxicos encontrados en sedimentos marinos existentes en la Bahía de Ite, Provincia de Jorge Basadre Grohmann de Tacna, en los recursos marinos bentónicos de la zona.

Determinar los parámetros oceanográficos en la zona de estudio y su incidencia en la fauna marina bentónica.

\section{DESARROLLO EXPERIMENTAL}

\subsection{Ubicación de la Zona de Estudio:}

El área de interés en la cual se consideró efectuar un estudio de los niveles de metales ecotóxicos en sedimentos superficiales está ubicada en el Distrito de Ite, Provincia de Jorge Basadre Grohmann de Tacna, Bahía de Ite. Por el norte el área de estudio alcanzará hasta Punta Alfarillo, y por el sur hasta las cercanías de Meca Grande, en la provincia de Jorge Basadre, lo que se muestra en la figura $\mathrm{N}^{\circ} 01$.

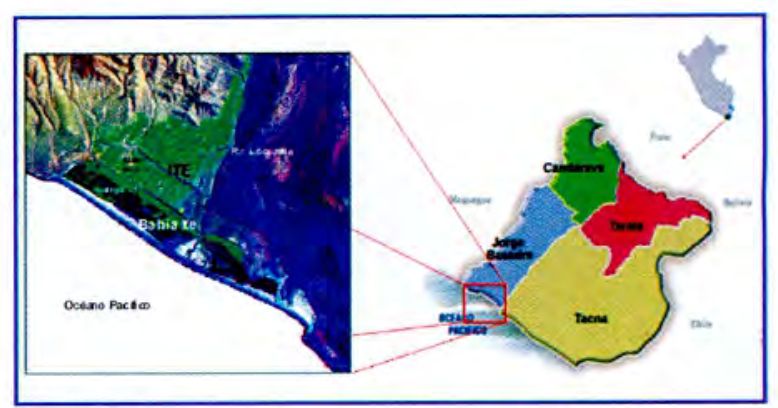

Figura Nº1: Ubicación del Área de Estudio (Elaboración propia)

\subsection{Diseño Experimental}

El periodo de muestreo se realizó entre el 17 y 20 de Julio del 2010, tomando en consideración aspectos oceanográficos y dinámica del mar, que permitan efectuar el muestreo en forma adecuada. En la Zona de estudio de la Bahía de Ite (Estaciones de muestreo E-1 a E-9) ubicadas en la zona considerada contaminada, se colectaron muestras de sedimentos marinos, analizando en estos además de los elementos ecotóxicos, la cuantificación de la fauna marina bentónica de cada estación, a lo largo de 3 perfiles con un total de nueve puntos de muestreo (figura $\mathrm{N}^{0} 02$ ), para la toma de muestras para el análisis de trazas de metales se utilizó la técnica de dragado.

Una vez obtenidas las muestras de sedimentos, se codificaron y colocaron en cajas térmicas para su preservación con hielo, este tipo de tratamiento se realizó en cada estación. El análisis de metales ecotóxicos de realizó en el laboratorio certificado Inspectorate Peru $\mathrm{SaC}$, de la capital de la republica y la cuantificación de la fauna marina bentónica se realizó en el Laboratorio Regional del Instituto del Mar de Ilo.

\subsection{Bahía de Ite}

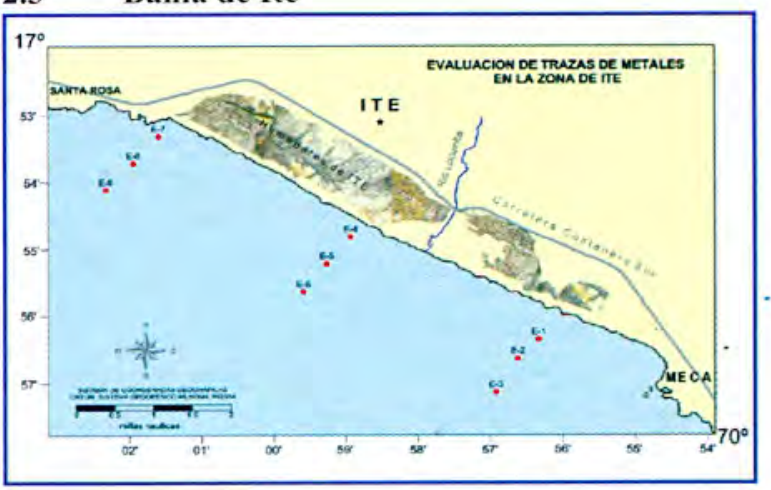

Figura $\mathrm{N}^{\circ}$ 02: Distribución geográfica de las nueve estaciones de muestreo, ubicadas en la Bahía de Ite, (zona de estudio) en tres perfiles de tres puntos de muestreo por perfil. 
Se ubica al Sur del Perú en la Región Tacna, Provincia de Jorge Basadre, Distrito de Ite, lugar denominado Playa Inglesa (Figura $\mathrm{N}^{\circ} 02$ ), formada por una playa artificial de relaves mineros, la zona cuenta con vegetación, formando los denominados humedales de Ite, no existen en el lugar descargas antropogénicas de origen doméstico. El agua que alimenta los humedales proviene de las filtraciones de la represa de Quebrada Honda, donde se almacenan en la actualidad los relaves mineros que generan las minas de Cuajone y Toquepala.

\section{RESULTADOS}

\subsection{Resultados Metales Ecotóxicos en la Bahía de Ite}

Tabla N ${ }^{\circ} 01$ Estadística Descriptiva de las variables medidas en los Sedimentos marinos de Bahía de Ite

\begin{tabular}{|l|c|r|r|r|r|}
\hline \multicolumn{1}{|c|}{ Variable } & $\begin{array}{c}N^{\circ} \\
\text { Datos }\end{array}$ & \multicolumn{1}{c|}{ Media } & \multicolumn{1}{c|}{ DS } & Minmo & \multicolumn{1}{c|}{ Máximo } \\
\hline Cobre $(\mathrm{ma} / \mathrm{ka})$ & 9 & 608.063 & 196.694 & 288.430 & 935.140 \\
\hline Znc $(\mathrm{ma} / \mathrm{ka})$ & 9 & 36.923 & 5.075 & 28.500 & 44.630 \\
\hline Arsénico $(\mathrm{ma} / \mathrm{ka})$ & 9 & 8.660 & 1.946 & 6.010 & 11.040 \\
\hline Cadmio $(\mathrm{ma} / \mathrm{ka})$ & 9 & 0.410 & 0.184 & 0.220 & 0.720 \\
\hline Mercurio $(\mathrm{ma} / \mathrm{ka})$ & 9 & 0.010 & 0.000 & 0.010 & 0.010 \\
\hline Plomo $(\mathrm{ma} / \mathrm{ka})$ & 9 & 8.472 & 1.297 & 6.180 & 10.500 \\
\hline Hierro $(\mathrm{ma} / \mathrm{ka})$ & 9 & 33078.633 & 19998.774 & 14596.370 & 81106.980 \\
\hline
\end{tabular}

\subsection{Resultados de Humedad, pH y Materia Orgánica en Sedimentos}

Los resultados del porcentaje de humedad, potencial de hidrógeno y materia orgánica en la Bahía de Ite en las nueve estaciones de muestreo se muestran en la tabla $\mathrm{N}^{\circ} 02$.

Tabla $\mathbf{N}^{0}$ 02: Porcentaje de humedad, potencial de hidrógeno y materia orgánica en la Bahía de Ite

\begin{tabular}{|c|c|c|c|c|}
\hline $\begin{array}{c}\text { Código de } \\
\text { Laboratorio }\end{array}$ & $\begin{array}{c}\text { Estación de } \\
\text { Muestreo }\end{array}$ & $\begin{array}{c}\text { Humedad } \\
\%\end{array}$ & $\begin{array}{c}\mathrm{pH} \\
\text { Unidades }\end{array}$ & $\begin{array}{c}\text { Materia } \\
\text { Orgánica \% }\end{array}$ \\
\hline $22192-122188$ & $\mathrm{E}-1$ & 25,2 & 7,6 & 0,37 \\
\hline $2192-122189$ & $\mathrm{E}-2$ & 25,9 & 7,5 & 0,56 \\
\hline $22192-122190$ & $\mathrm{E}-3$ & 26 & 7,7 & 0,74 \\
\hline $22192-122191$ & $\mathrm{E}-4$ & 23,3 & 74 & 0.39 \\
\hline $22192-122192$ & $\mathrm{E}-5$ & 25,9 & 7.6 & 0.68 \\
\hline $2192-122193$ & $\mathrm{E}-6$ & 24,9 & 7,4 & 0,55 \\
\hline $22192-122194$ & $\mathrm{E}-7$ & 23,5 & 7,4 & 0,35 \\
\hline $22192-122195$ & $\mathrm{E}-8$ & 24.9 & 7.7 & 0.61 \\
\hline $22192-122196$ & $\mathrm{E}-9$ & 24.5 & 7.8 & 0.46 \\
\hline
\end{tabular}

3.3 Resultados del Análisis de la Comunidad bentónica Bahía de Ite

Se analizaron muestras de macrobentos de las 09 estaciones de muestreo, entre 10,2 y 21,9 metros de profundidad. Se realizó un análisis cualitativo y cuantitativo, utilizando tanto el número de especies, número de ejemplares de cada especie y biomasa; también se consideró el área de influencia de las principales fuentes contaminantes en la Bahía de Ite y determinar en qué medida la composición de estas especies se ve afectada por los aportes antropogénicos.

Tabla $\mathrm{N}^{\circ}$ 03: Número de ejemplares de cada especie, por estación de muestreo.

\begin{tabular}{|c|c|c|c|c|c|c|c|c|c|c|c|c|}
\hline \multirow[t]{2}{*}{ TAXA } & \multicolumn{3}{|c|}{ Centro } & \multirow{2}{*}{$\begin{array}{l}\text { Total } \\
\text { centro }\end{array}$} & \multicolumn{2}{|c|}{ Norte } & \multirow{2}{*}{$\begin{array}{l}\text { Total } \\
\text { norte }\end{array}$} & \multicolumn{3}{|c|}{ Sur } & \multirow{2}{*}{$\begin{array}{c}\text { Total } \\
\text { s ur }\end{array}$} & \multirow{2}{*}{$\begin{array}{c}\text { Total } \\
\text { ge ne ral }\end{array}$} \\
\hline & E-4 & E-5 & E-6 & & E-8 & E-9 & & E-1 & E-2 & E-3 & & \\
\hline Annelida & 4 & 137 & 42 & 183 & 18 & 22 & 40 & 4 & 21 & 41 & 66 & 289 \\
\hline \multicolumn{13}{|l|}{ Cirratulidae } \\
\hline Diopatra sp. & & & & & & 1 & 1 & & 1 & & 1 & 2 \\
\hline Glycera sp. & & & 12 & 12 & 4 & 5 & 9 & & 8 & & 8 & 29 \\
\hline Nepthys sp. & 4 & 98 & 26 & 128 & 14 & 12 & 26 & 4 & 12 & 40 & 56 & 210 \\
\hline \multicolumn{13}{|l|}{ Orbinia sp. } \\
\hline Phylodoce sp. & & 39 & 2 & 41 & & & & & & 1 & 1 & 42 \\
\hline \multicolumn{13}{|l|}{ Syllis sp. } \\
\hline Arthropoda & 2. & 10 & 3 & 15 & 1 & 7 & 8 & & & 11 & 11 & 34 \\
\hline Gamm aridea & & 10 & & 10 & 1 & 6 & 7 & & & 10 & 10 & 27 \\
\hline \multicolumn{13}{|l|}{ Isocheles pacificus } \\
\hline \multicolumn{13}{|l|}{ Ogyridae } \\
\hline Pseudocorys thes sicarius & 1 & & 1 & 2 & & 1 & 1 & & & 1 & 1 & 4 \\
\hline Cnidaria & & & 26 & 2.6 & & 10 & 10 & & 7 & & 7 & 43 \\
\hline Actini sp. & & & 26 & 26 & & 10 & 10 & & 7 & & 7 & 43 \\
\hline Mollusca & & 3 & & 3 & & 1 & 1 & & 2 & & 2 & 6 \\
\hline \multicolumn{13}{|l|}{ Nassarius gavi } \\
\hline \multicolumn{13}{|l|}{ Policines uber } \\
\hline Semimytilus algosus & & 3 & & 3 & & & & & & & & 3 \\
\hline \multicolumn{13}{|l|}{ Nematoda. } \\
\hline \multicolumn{13}{|l|}{ Nematodo } \\
\hline Total ge ne ral & 6 & 150 & 71 & 227 & 19 & 40 & 59 & 6 & 30 & 52 & 88 & 374 \\
\hline Tetal generaL Biomasa & 3.21 & 1.9 & 7.21 & 12.28 & 0.21 & 7.1 & 7.2 .6 & 0.08 & 2 & 4.4 & $6,4,2$ & 25.96 \\
\hline
\end{tabular}

Mediante la utilización del software estadístico PRIMER V:6,0 (Clarke \& Warwick 1994; Clarke \& Gorley 2001) se obtuvo los índices ecológicos como el Predominio de Simpson ( $\lambda$ ), el Predominio de Hill (N1 y N2), la Uniformidad de Pielou`s (J), la Equidad de Hill (N21), la Diversidad de Hill (N10) y la Diversidad de Shannon ( $\left.\mathrm{H}^{\prime}\right)$ que utiliza la riqueza de especies y la equitatividad. 
Tabla Nº4: Biomasa de las especies por estación de muestreo: Bahía de Ite

\begin{tabular}{|c|c|c|c|c|c|c|c|c|c|c|c|c|}
\hline \multirow{2}{*}{ Rótulos de fila } & \multicolumn{3}{|c|}{ Centro } & \multirow{2}{*}{$\begin{array}{c}\text { Total } \\
\text { centro }\end{array}$} & \multicolumn{2}{|c|}{ Norte } & \multirow{2}{*}{$\begin{array}{c}\text { Total } \\
\text { norte }\end{array}$} & \multicolumn{3}{|c|}{ Sur } & \multirow{2}{*}{$\begin{array}{c}\text { Total } \\
\text { sur }\end{array}$} & \multirow{2}{*}{$\begin{array}{r}\text { Total } \\
\text { genera }\end{array}$} \\
\hline & $E-4$ & $E-5$ & $E-6$ & & $E-8$ & E.9 & & E-1 & $E-2$ & $E-3$ & & \\
\hline Actin ia sp. & & & 3.07 & 3.07 & & 1.11 & 1.11 & & 1.57 & & 1.57 & 5.75 \\
\hline Cirratulidae & & & & & & 0.01 & 0.01 & & & & & 0.01 \\
\hline Diopatra sp. & & & & & & 0.01 & 0.01 & & 0.03 & & 0.03 & 0.04 \\
\hline Gamm aride a & & 0.01 & & 0.01 & 0.01 & 0.01 & 0.02 & & & 0.01 & 0.01 & 0.04 \\
\hline Glyce ra sp. & & & 0.08 & 0.08 & 0.13 & 0.02 & 0.15 & & 0.16 & & 0.16 & 0.39 \\
\hline Isocheles pacificus & 0.67 & & & 0.67 & & & & & & & & 0.67 \\
\hline Nas sarius gayi & & & & & & & & & 0.05 & & 0.05 & 0.05 \\
\hline Nemato do & & & & & & & & 0.02 & & & 0.02 & 0.02 \\
\hline Nepthys sp. & 0.07 & 0.88 & 0.3 & 1.25 & 0.07 & 0.09 & 0.16 & 0.06 & 0.14 & 0.48 & 0.68 & 2.09 \\
\hline Ogyridae & & & 0.15 & 0.15 & & & & & & & & 0.15 \\
\hline Orbinia sp. & & & & & & 0.01 & 0.01 & & & & & 0.01 \\
\hline Phyllod oce sp. & & 0.97 & 0.02 & 0.99 & & & & & & 0.01 & 0.01 & 1 \\
\hline Policines uber & & & & & & 3.13 & 3.13 & & & & & 3.13 \\
\hline Ps eud ocorysthes sicarius & 2.47 & & 3.52 & 5.99 & & 2.66 & 2.66 & & & 3.89 & 3.89 & 12.54 \\
\hline Sem im ytilus al gosus & & 0.06 & & 0.06 & & & & & & & & 0.06 \\
\hline Syllis sp. & & & 0.01 & 0.01 & & & & & & & & 0.01 \\
\hline Total general & 3.21 & 1.92 & 7.15 & 12.28 & 0.21 & 7.05 & 7.26 & 0.08 & 1.95 & 4.39 & 6.42 & 25.96 \\
\hline
\end{tabular}

Tabla Nº5: Promedio de los Índices ecológicos (Uniformidad, Predominio, Diversidad, Riqueza de especies por sector-Bahía Ite

\begin{tabular}{|c|c|c|c|c|c|c|c|c|}
\hline \multirow{3}{*}{$\begin{array}{l}\text { Índices } \\
\text { ecológicos }\end{array}$} & \multicolumn{8}{|c|}{ Sectores de muestreo en la zona "Ite" } \\
\hline & \multicolumn{3}{|c|}{ Centro } & \multicolumn{2}{|c|}{ Norte } & \multicolumn{3}{|c|}{ Sur } \\
\hline & $E-4$ & $E-6$ & $E-5$ & $E-9$ & $E-8$ & $E-1$ & $E-2$ & $E-3$ \\
\hline Especies (s) & 3 & 7 & 4 & 9 & 3 & 2 & 5 & 4 \\
\hline Individuos & 6 & 71 & 150 & 40 & 19 & 6 & 30 & 52 \\
\hline Margalef (d) & 1,116 & 1,408 & 0,599 & 2,169 & 0,679 & 0,558 & 1,176 & 0,759 \\
\hline Pielou's (J') & 0,79 & 0,718 & 0,64 & 0,832 & 0,644 & 0,918 & 0,84 & 0,484 \\
\hline Shannon $\left(\mathrm{H}^{\prime}\right)$ & 1,252 & 2,017 & 1,28 & 2,638 & 1,021 & 0,918 & 1,951 & 0,968 \\
\hline Simpson $(\lambda)$ & 0,5 & 0,299 & 0,499 & 0,198 & 0,59 & 0,556 & 0,291 & 0,629 \\
\hline Hill (N1) & 2,381 & 4,047 & 2,428 & 6,225 & 2,03 & 1,89 & 3,867 & 1,956 \\
\hline Hill (N2) & 2 & 3,341 & 2,003 & 5,063 & 1,695 & 1,8 & 3,435 & 1,589 \\
\hline Hill (N10) & 0,794 & 0,578 & 0,607 & 0,692 & 0,677 & 0,945 & 0,773 & 0,489 \\
\hline Hill (N21) & 0,84 & 0,826 & 0,825 & 0,813 & 0,835 & 0,952 & 0,888 & 0,812 \\
\hline$\%$ que representa $\mathrm{N} 1$ & 79.4 & 57.8 & 60.7 & 69.2 & 67.7 & 94.5 & 77.3 & 48.9 \\
\hline$\%$ que representa N2 & 66.7 & 47.7 & 50.1 & 56.3 & 56.5 & 90.0 & 68.7 & 39.7 \\
\hline
\end{tabular}

\section{3,4 Resultados muestreo oceanográfico Bahía de Ite}

En la Tabla $\mathrm{N}^{\circ} 06$ presentamos la ubicación de las estaciones de muestreo, fecha y hora, profundidad a la que fue tomada la muestra, y resultados de temperatura del agua, oxígeno disuelto en el lugar de la toma de la muestra, salinidad y transparencia del agua, para el muestreo oceanográfico se consideraron nueve estaciones.

Tabla $\mathbf{N}^{\circ}$ 06: Ubicación y resultados del muestreo oceanográfico

\begin{tabular}{|c|c|c|c|c|c|c|c|c|c|c|}
\hline \multirow{2}{*}{$\mathrm{N}^{\circ}$} & \multirow{2}{*}{ Estación } & \multicolumn{3}{|c|}{ Fecha } & \multirow{2}{*}{ Hora } & \multirow{2}{*}{$\begin{array}{l}\text { P r of un di } \\
\text { dad }(m)\end{array}$} & \multirow{2}{*}{$\begin{array}{c}\text { T em pe } \\
\text { ratur a } \\
\left({ }^{\circ} \mathrm{C}\right)\end{array}$} & \multirow{2}{*}{$\begin{array}{c}\text { Oxígeno } \\
(m L / L \text {.) }\end{array}$} & \multirow{2}{*}{$\begin{array}{l}\text { Salin idad } \\
\text { (UPS) }\end{array}$} & \multirow{2}{*}{$\begin{array}{c}\text { Transpa } \\
\text { rencia } \\
\text { (m) }\end{array}$} \\
\hline & & A ño & Mes & Día & & & & & & \\
\hline 1 & E-1 & 2010 & 7 & 20 & $12: 00$ & 0 & 14,3 & 4 & 34,728 & 3 \\
\hline 2 & & & & & & 11,9 & 14,1 & 3,78 & 34,723 & \\
\hline 3 & E-2 & 2010 & 7 & 20 & $11: 45$ & 0 & 14,3 & 3,89 & 34,750 & 5 \\
\hline 4 & & & & & & 17,8 & 13,7 & 2,28 & 34,738 & \\
\hline 5 & E-3 & 2010 & 7 & 20 & $11: 35$ & 0 & 14,3 & 3,81 & 34,764 & 8 \\
\hline 6 & & & & & & 21,9 & 13,6 & 0,34 & 34,734 & \\
\hline 7 & E-4 & 2010 & 7 & 20 & $10: 55$ & 0 & 14,4 & 4,27 & 34,736 & 5 \\
\hline 8 & & & & & & 10,2 & 14,2 & 3,87 & 34,734 & \\
\hline 9 & E-5 & 2010 & 7 & 20 & $10: 40$ & 0 & 14,4 & 3,95 & 34,744 & 8 \\
\hline 10 & & & & & & 15,1 & 13,3 & 2,56 & 34,740 & \\
\hline 11 & $\mathrm{E}-6$ & 2010 & 7 & 20 & $10: 20$ & 0 & 14,4 & 3,24 & 34,749 & 10 \\
\hline 12 & & & & & & 17,7 & 13,8 & 8,86 & 34,764 & \\
\hline 13 & E-7 & 2010 & 7 & 17 & $10: 26$ & 0 & 15,2 & 4,7 & 34,732 & 1.5 \\
\hline 14 & & & & & & 11 & 14,6 & 3,11 & 34,730 & \\
\hline 15 & E-8 & 2010 & 7 & 17 & $10: 09$ & 0 & 15,2 & 3,88 & 34,746 & 5 \\
\hline 16 & & & & & & 15,9 & 14,4 & 1,91 & 34,737 & \\
\hline 17 & E-9 & 2010 & 7 & 17 & $9: 51$ & 0 & 15,3 & 3,77 & 34,792 & 5 \\
\hline 18 & & & & & & 19,7 & 14,7 & 0,52 & 34,740 & \\
\hline
\end{tabular}




\section{DISCUSIÓN DE RESULTADOS}

\subsection{Discusión de resultados de la comunidad bentónica en la Bahía de Ite}

La contaminación por metales ecotóxicos constituye un grave problema en la Bahía de Ite. Una de las consecuencias de ello es la modificación de los sedimentos por los relaves mineros (Dames y Moore 1976), los cuales forman una barrera que impide el normal proceso de oxigenación entre la columna de agua y los sedimentos naturales (Orozco et al., 1998).

La Bahía de Ite, presento una abundancia que osciló entre 6 y $150 \mathrm{ind} / 0,05 \mathrm{~m}^{2}$ (Tabla $\mathrm{N}^{\circ} 03$ ), y una biomasa entre 0,08 y $7,15 \mathrm{~g} / 0,05 \mathrm{~m}^{2}$. Orozco et al., 1998, reporta bajos valores de abundancia y biomasa en esta Bahia, principalmente por la modificación en la estructura del tipo de sustrato, generando un cambio gradual en la distribución y presencia de los organismos bentónicos; por otro lado, es importante mencionar que estos cambios que afectan directamente la estructura de las comunidades también favorecen la proliferación de cierto grupo de especies, destacando entre ellos las especies de poliquetos Nepthys sp y Glycera sp.

También es importante mencionar la presencia de los crustáceos Pseudocorysthes sicarius y las especies del grupo Gammaridea, además de las especies de los phyla; Cnidaria, Mollusca y Nematoda

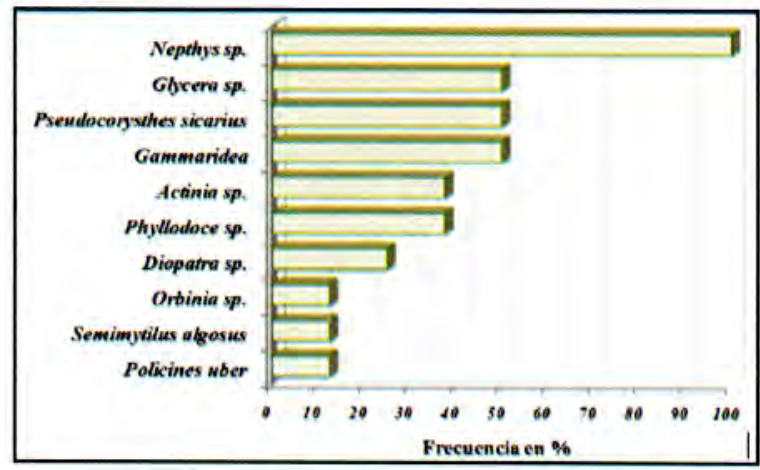

Figura $\mathbf{N}^{\mathbf{0}}$ 03: Porcentaje de frecuencia de las principales especies de la macro-infauna en la zona de Ite

Según los resultados mostrados en la tabla $\mathrm{N}^{\circ} 05$ la riqueza especifica de Margalef (d) osciló entre 0,558 y 2,169 lo que permite catalogar como un ambiente bajo en diversidad.

El índice de diversidad de Shannon $\left(\mathrm{H}^{\prime}\right)$, reportó valores entre 0,918 y 2,638 bits/ind, encontrándose estos resultados, en su mayoría, en el nivel de poca representatividad entre $(1,00-2,00)$ por tanto estaciones con diversidades poco representativas, generado principalmente por actividades antropogénicas.

El indice de Simpson $(\lambda)$, manifiesta la probabilidad de que dos individuos tomados al azar de una muestra sean de la misma especie. Está fuertemente influido por la importancia de las especies más dominantes. El índice de Simpson $(\lambda)$ en nuestro estudio presentó una variación de 0,198 (E-9) a 0,629 (E-3). Estos valores se encuentran dentro del rango de baja y mediana dominancia por parte de alguna de las especies.

El índice de equitabilidad de Pielou's (J'); representa cuan equitativa están distribuidas las especies en una determinada zona; en ella se mide la proporción de la diversidad observada con relación a la máxima diversidad esperada. Su valor va de 0,0 a 1,0. En la Bahía de Ite se reportan indices de equitabilidad que varían desde 0,484 (E-3) hasta valores 0,918 (E-1). Esta relación nos permite establecer la presencia de estaciones con poca uniformidad y/o equitativilidad dentro de un mismo sector, como es el caso del sector sur donde la mayor uniformidad se presentó en la estación más somera (E-1) y la menos uniforme en la estación más profunda (E-3).

Los indices de Hill (N1 y N2); que nos determinan la presencia de especies abundantes (N1) y especies muy abundantes (N2) fueron altos en la mayoría de las estaciones sobre todo en E-6, E-9 y E-2, donde los porcentajes de especies abundantes y muy abundantes fueron superior al $50 \%$ del total identificado.

Los valores de diversidad de Hill (N10); se enmarca dentro de la escala de 0,0 (altamente diverso) a 1,0 (menos diverso), y que según nuestros resultados la mayoría de estaciones presentaron valores superiores a 0,6 por tanto se pueden interpretar como estaciones con poco representativos en diversidad. Finalmente el índice de equidad de Hill (N21), oscilo entre 0,812 (E-3) y 0,952 (E-1), lo que la coloca como ambientes poco uniformes por presentar valores cercanos a 1,0 dentro de la escala de clasificación.

\subsubsection{Perturbación comunitaria: Curvas $A B C$ (Abundace/Biomass Comparison) Warwick et al., 1987}

Mediante el análisis del K-dominancia de Lambshead et al., 1983 se obtuvo el porcentaje acumulado de la abundancia y biomasa graficando las curvas $A B C$ en cada sector de la zona de Ite y así poder determinar el grado de perturbación y/o cambios en la estructuración del ambiente submareal de la mencionada zona.

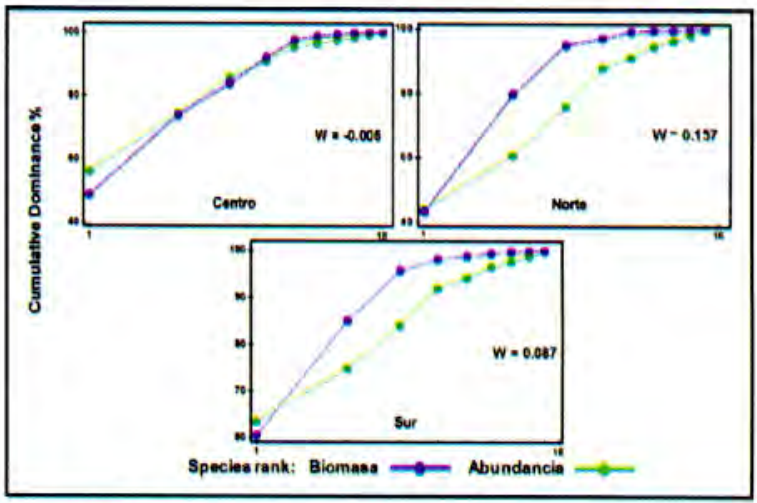

Figura $N^{\circ}$ 04: Curvas del $\mathrm{ABC}$ (Abundace/Biomass Comparison) según lo indica Warwick (1986), por sector: centro, norte y sur - Ite 
En función a ello podemos notar que en cada uno de los sectores la tendencia de perturbación fue diferente, es así que en el sector centro la curva de biomasa se situó por debajo de la abundancia con porcentajes acumulados menores al $50 \%$ por tanto un ambiente catalogado como perturbado $(\mathrm{P})$. Por otro lado en el sector sur la curva de biomasa presentó un ligero entrecruzamiento con la curva de abundancia por tanto catalogada con moderada o leve perturbación (MP); finalmente en el sector norte la curva de biomasa se situó por encima de la abundancia permitiendo su catalogación como un ambiente no perturbado (NP).

Esta tendencia también se ve reflejada por los valores del estadístico "W" donde el sector centro fue negativo $(-0.006)$, sector sur positivo pero cercano a cero $(0.087)$ mientras que el sector norte fue positivo $(0.157)$ (Figura $\left.\mathrm{N}^{\circ} 04\right)$.

De las 16 especies de invertebrados capturados, destacaron los poliquetos Nepthys sp., Phyllodoce sp. $y$ Glycera $s p$. además de las especies actinias y los crustáceos del grupo Gammaridea (Figura $\mathrm{N}^{\circ} 05$ ) por presentar los más altos niveles de abundancia en toda el área estudiada, mientras que las especies Nepthys $s p$., Glycera sp, Pseudocoristhes sicarius y Gammaridea entre otras destacaron por haber sido registrado en más del $50 \%$ de los muestreos ejecutados.

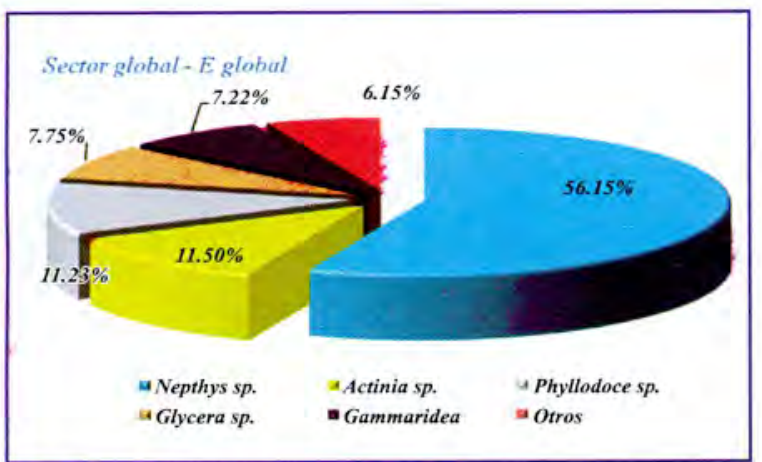

Figura $\mathbf{N}^{\mathbf{0} 05}$ : Porcentajes de abundancia de las principales 5 especies de la macro-infauna en la zona de Ite

\subsection{Discusión de Resultados Oceanográficos}

\section{Temperatura $\left({ }^{\circ} \mathrm{C}\right)$}

La Bahía de Ite, presentó condiciones térmicas frías, donde los valores de temperatura a nivel superficial disminuyeron hacia el sur, variando de 14,3 a $15,3{ }^{\circ} \mathrm{C}$. La temperatura a nivel de fondo fluctuó de 13,3 a 14,7 ${ }^{\circ} \mathrm{C}$, en profundidades que varían de 10,2 a $21,9 \mathrm{~m}$, incrementándose la estructura térmica cerca a la línea de playa y hacia el norte de la zona de estudio. (Figura $\mathrm{N}^{\circ} 01$ y Tabla No6). El Instituto del Mar del Perú, Estación Costera Ilo, reporta para el mes de Julio un promedio de los años $1996-2010$, de $15,5{ }^{\circ} \mathrm{C}$, efectuando la comparación de los valores promedios reportados en nuestro estudio, indicaríamos que están dentro del rango normal de temperatura. La Bahía de Ite y Morro Sama, se considera zona comúnmente fría por efectos de afloramientos costero (Cabello et al., 2000).

\section{$1 \quad$ Salinidad (UPS)}

Como se muestra en la Figura $\mathrm{N}^{\circ} 01$, los resultados de la Tabla $\mathrm{N}^{\circ} 06$, la distribución de las isohalinas superficiales variaron entre 34,728 a 34,792 UPS y los valores de la salinidad a nivel profundo fluctuaron entre 34,723 a 34,764 UPS, nos muestra la influencia de las corrientes frías Sub Antárticas en la columna de agua tanto en superficie como en profundidad en el área evaluada. Los valores presentados son valores propios de aguas costeras frías. Según la Estación Costera del Instituto del Mar del Perú del Puerto de Ilo, reporta un promedio de salinidad para el mes de Julio de los años 1996 -2010, de 34,864 UPS; en consecuencia los datos reportados por nuestro estudio estarían en el rango normal. Los valores ligeramente bajos podrían ser causa del efecto de la niña en la época de muestreo.

\section{A Oxígeno $(\mathrm{mL} / \mathrm{L})$}

El contenido de oxígeno en agua marina y sedimentos marinos, puede ser alterado por diferentes procesos bióticos y abióticos, siendo el de mayor relevancia el proceso abiótico de descomposición de la materia orgánica. Esto se debe a la acción bacteriana de microorganismos sobre el material fresco, los cuales utilizan como fuente potencial el oxígeno y la energía química producida en el mecanismo de mineralización para su metabolismo (Bonilla et al., 2003).

Analizando la concentración de oxígeno superficial en el agua de mar del área de estudio presentó valores de 3,24 a $4,70 \mathrm{~mL} / \mathrm{L}$, y a nivel de fondo el rango varió de 0,43 a $3,87 \mathrm{~mL} / \mathrm{L}$; lo cual indica que va incrementando hacia la línea de costa. Estos valores de oxígeno también pueden ser consecuencia de la presencia de las aguas Sub Antárticas que se caracterizan por tener bajas concentraciones de oxígeno disuelto. En consecuencia los valores de oxígeno disuelto en el agua de mar son valores propios de aguas costeras frías (Sánchez et al 1998).

\section{$\Delta \quad$ Transparencia (m)}

La transparencia del agua de mar en el área de estudio, presentó valores de 1,5 a $10,0 \mathrm{~m}$, relacionándose con la distribución del oxígeno, indicándonos que las mayores concentraciones del oxígeno y la alta productividad (menor transparencia) se producen generalmente en las áreas más costeras.

\section{CONCLUSIONES}

1. El impacto de la contaminación sobre la fauna bentónica sigue siendo grave en la Bahía de Ite, con puntos críticos en la zona central, de acuerdo a los índices ecológicos obtenidos para toda la Bahía, se puede establecer que la comunidad bentónica distribuida en la zona de influencia de la descarga de los relaves se encuentra perturbada ecológicamente, además mostro muy pobre riqueza específica, encontrándose a los poliquetos como las especies más representativos, entre ellas la especie Nepthys sp., Phyllodoce sp. y Glycera sp. Lo que nos da la certeza que la zona esta alterada ecológicamente. 
2. Los condiciones oceanográficas; temperatura, salinidad, oxigeno disuelto, transparencia, presentaron valores uniformes y normales a nivel superficial y de fondo; no se aprecia notable variación con datos promedios reportados para el mes de julio por el Instituto del Mar del Perú, Estación Costera de Ilo.

\section{REFERENCIAS BIBLIOGRÁFICAS}

Ahumada, R. (1994). Herramientas conceptuales para la conservación del ambiente costero: criterios para evaluar los impactos ambientales en los sistemas marinos. Tercer curso de Contaminación Marina: "Marco Conceptual y Criterios Científicos- Técnicos para Establecer normas de emisión de Contaminantes". Comité Oceanográfico Nacional, CONA.

Bonilla, J. Aranda, S. Ramírez, C. Moya, J. Márquez A. 2003. Calidad de los Sedimentos Superficiales de la Ensenada Grande del Obispo, Estado Sucre -Venezuela. Instituto Oceanográfico de Venezuela, Universidad del Oriente, Cumaná, Venezuela.

Cabello, Rita; Jacinto, María; Morón, Octavio; Villanueva, Patricia. 2000. Calidad Acuática del Ambiente Marino Costero en las Áreas de Ilo e Ite, Noviembre 1997. IMARPE, Lima.

Dames y Moore 1976. Marine Environmental studies tailings discharge, Ite. Perú. Southern Perú Cooper Corporation. New York.

Harper, J. L. Y D. L. Hawksworth. 1994. Biodiversity: measurement and estimation (preface). Philosophical Transactions of the Royal Society of London Series B, 345: 5-12.
Hurlbert, S. H. 1971. The nonconcept of species diversity: a critique and alternative parameters. Ecology, 52: 577-586.

Magurran, A. E. 1988. Ecological diversity and its measurement. Princeton University Press, New Jersey, 179 pp.

Orozco, Rita; Enríquez Edgardo; Castillo Sonia; Fernández Elizabeth; Morón Octavio. 1996. Contaminación sobre el macrobentos en las Bahias de Mollendo, Ilo e Ite, Marzo Agosto 1996. IMARPE, Lima.

Sánchez, G. y Zeballos J. 1988. Inventario de Fuentes de Contaminación Marina de origen terrestre; domésticos, industrials y mineros de Pisco, Ilo, Ite y Chimbote. Primera parte. Procede del Inventario de Fuentes de Contaminación del Pacífico Sudeste. Documento CPPS/UCR, Plan de Acción Pacífico Sudeste con A poyo del PNUMA, Bogota, Julio 1988.

Solbrig, O. T. 1991. From genes to ecosystems: a research agenda for biodiversity. IUBSSCOPEUNESCO, Cambridge, 124 pp.

Spellerberg, I. F. 1991. Monitoring ecological change. Cambridge University Press, UK, 334 pp.

\section{Correspondencia:}

Lorenzo Walter Ibárcena Fernández Ciudad Universitaria Fundo "Los Granados" Calle Miraflores s/n. Tacna. Perú. wibarcena@gmail.com 